\title{
Melanoma Risk Factor
}

National Cancer Institute

\section{Source}

National Cancer Institute. Melanoma Risk Factor. NCI Thesaurus. Code C156749.

Any factor, such as age, ethnicity, genetic factor, family history, or environmental factor, that increases a person's chance of developing melanoma. 